dysrhythmia during sleep. Seizures were resistant to ACTH and antiepileptic drugs. Screening for these mutations is recommended in children with intractable seizures and global developmental delay. (Elia M, Falco M, Ferri R, et al. CDKL5 mutations in boys with severe encephalopathy and early-onset intractable seizures. Neurology Sept 2008;71:997999). (Respond: Dr Maurizio, Oasi Institute for Research on Mental Retardation and Brain Aging, Via Conte Ruggero 73, 94018 Troina (EN), Italy).

COMMENT. CDKL5 mutations are reported in girls with $\mathrm{X}$-linked infantile spasms (Weaving LS et al, 2004) and in girls with atypical Rett syndrome (Evans JC et al, 2005). The association of CDKL5 mutations and seizures is rare in boys (Van Esch $\mathrm{H}$ et al, 2007).

The recognition of this genetic diagnosis would be helpful in counseling concerning outcome.

The role of genetics in diagnosis and treatment of epilepsy was discussed at the First North American Regional Epilepsy Congress; $60^{\text {th }}$ Annual Meeting of the American Epilepsy Society, Dec 1-5, 2006, San Diego, CA (Bebin M. In: Arnedo V et al. Rev Neurol Dis 2007;4(4):217-221). The nocturnal frontal lobe epilepsy gene, autosomal dominant with $75 \%$ penetrance, was one of the first identified. The gene mapped to chromosome $20 \mathrm{q}$ and the nicotinic receptor opened the door to the channelopathies. Voltage-gated ion channel subunits are involved in GEFS+, Dravet's syndrome, and infantile seizures; potassium channels in neonatal seizures; and potassium and calcium channels in absence epilepsy. $S C N 1 A$ gene controls the sodium channel subunit in Dravet's syndrome, febrile seizures, febrile seizures plus, and myoclonic-astatic epilepsy. SCN1A mutations occur in $79 \%$ of severe myoclonic epilepsies of infancy (SMEI) and $69 \%$ of borderline cases (Scheffer IE et al. Brain Dev 2001;23:732-735; idem. In: Arnedo V et al. Rev Neurol Dis 2007;4:217-221).

\title{
ATTENTION DEFICITS AND ROLANDIC EPILEPSY
}

Impairment in attention in rolandic epilepsy (RE) was evaluated in 14 studies published between 1990 and 2006, in a study at Columbia University Medical Center and Queens College of the City University of New York, NY. Sample sizes ranged from 9 to 44 subjects. A cross-sectional design was used in 7 studies ( 6 having active EEG abnormalities and 1 with EEG remission). Longitudinal studies were employed in 7 publications (EEGs were all abnormal and patients were followed until normalized). Twelve studies measured the alerting network, according to the Posner model of attention, 11 studies the orienting network, and 8 the executive network. Impairments in all attention networks were demonstrated by controlled studies but not by uncontrolled studies. At follow-up, when the EEG had normalized, attention impairments had almost completely resolved. The neuroanatomically based model of attention such as Posner's that targets alerting, orienting, and executive functions, is a reliable method of assessment of attention in children with epilepsy. (Kavros PM, Clarke T, Strug LJ, Halperin JM, Dorta NJ, Pal DK. Attention impairment in rolandic epilepsy: systematic review. Epilepsia Sept 2008;49(9):1570-1580). (Respond: Deb K Pal MD, PhD, Mailman School of Public Health, 722 West $68^{\text {th }}$ Street, Sixth Floor, New York, NY 10032. E-mail: dkp28'acolumbia.edu). 
COMMENT. Children with rolandic epilepsy are predisposed to impairments of attention that resolve with remission of EEG centrotemporal spikes. Attention impairment in RE is correlated not with seizures but with EEG epileptiform discharges. Children with RE should be monitored for attention, language, and learning disorders. Rolandic spikes may aggravate the course of ADHD and predispose to increased impulsivity (Holtmann M et al. Brain Dev 2006;28:633-640).

\section{ANXIETY AND DEPRESSION WITH FIRST SEIZURE}

Anxiety and depressive signs were investigated in 22 children (mean age, 12+/-2.7 years SD) with a first unprovoked afebrile seizure, in a study at Alberta Children's Hospital, University of Calgary, Canada. Compared with published norms for a Child Anxiety Scale and Depression Inventory, patients reported significantly higher scores for total anxiety, worry/oversensitivity, and social concerns/concentration. Compared with a medical cohort, anxiety symptoms were similar. Children with a first seizure had greater interpersonal problems, ineffectiveness, and negative self-esteem than published norms. Compared to controls, they had increased negative mood, ineffectiveness, anhedonia, and negative selfesteem. Anxiety may be related to illness or hospital experience, and depressive symptoms may be comorbid at time of seizure. (Loney JC, Wirrell EC, Sherman EMS, Hamiwka LD. Anxiety and depressive symptoms in children presenting with a first seizure. Pediatr Neurol Oct 2008;39:236-240). (Respond: Dr Hamiwka, Division of Pediatric Neurology, Alberta Children's Hospital, 2888 Shaganappi Trail NW, Calgary, Alberta T3B 6A8, Canada. Email: ihamiwka(a;mac.com).

COMMENT. Children presenting with a first seizure may be at increased risk of anxiety and depressive symptoms.

\section{DEMYELINATING DISORDERS}

\section{RISK FACTORS FOR MULTIPLE SCLEROSIS AFTER FIRST ATT ACK OF INFLAMMATORY CNS DEMYELINATION}

Clinical, radiologic, or CSF factors predicting development of multiple sclerosis (MS) after a first inflammatory demyelinating attack were identified in 117 children $(56 \%<10$ years, $34 \%<6$ years) participating in a nationwide retrospective multicenter study in the Netherlands. A second MS-defining attack occurred in $43 \%$ of 54 children who presented with a monofocal clinically isolated syndrome (CIS), compared to $21 \%$ of 63 patients with a polyfocal CIS $(\mathrm{p}<0.006)$. Lesions considered typical of ADEM (basal ganglia and thalamic lesions and lesions $>2 \mathrm{~cm}$ on MRI) occurred during PCIS, with or without encephalopathy. Children with PCIS had a preceding infection in $50 \%$, fever in $42 \%$, and seizures in $28 \%$; they were not at greater risk of developing MS. Children with PCIS without encephalopathy progressed to MS more frequently than those with encephalopathy (ADEM). Risk factors for development of MS included elevated IgG index, oligoclonal CSF bands, 3 Barkhof MRI criteria ( $>9$ lesions T2, infratentorial, juxtacortical, and $>3$ perivascular lesions), and KIDMUS supplemental MRI criteria (perpendicular to corpus callosum, thalamic/basal 